\title{
SOME ASPECTS OF AQUEOUS HUMOUR DRAINAGE
}

\author{
A. BILL \\ Uppsala, Sweden
}

\begin{abstract}
SUMMARY
It is now 50 years since the discovery of the aqueous veins by Karl Ascher. His finding had a great impact on ophthalmology since it showed that the aqueous humour is not a stagnant fluid; there had to be a continuous formation of aqueous. humour by the ciliary processes, flow from the posterior chamber into the anterior chamber and outflow in the chamber angle. Since that time it has become clear that there is also some drainage of aqueous humour via uveoscleral routes. Furthermore the flow through the inner wall of Schlemm's canal has been shown to take place through about 20000 pores each with diameter of around 0.1-3 $\mu \mathrm{m}$. Recent measurements of the pressures in the outflow routes indicate that in normal monkey eyes the main outflow resistance is located close to the inner wall of the canal. Most of the aqueous humour leaving Schlemm's canal via the collector channels mixes with blood within the sclera. This is a consequence of the embryology of Schlemm's canal; it develops by the merging of blind extensions from intrascleral veins. Despite its specialisation the endothelium of Schlemm's canal retains the properties of a blood vessel. Platelets are likely to play in role in the integrity of Schlemm's canal as they do in blood vessels and may in fact control the size of the pores by occluding pores larger than $3 \mu \mathrm{m}$. It seems likely that in vitro perfusion of glaucomatous eyes with enzymes may be useful in the search for new methods of treatment for glaucoma.
\end{abstract}

It is now 50 years since Karl Ascher first reported a simple observation which caused a fundamental change in ophthalmology, namely that vessels in the limbus region appeared to drain aqueous humour into the veins of the conjunctiva and the episclera. ${ }^{1,2}$ This observation made it clear that there is a continuous drainage of aqueous humour from the anterior chamber and that there has to be a corresponding inflow from the posterior chamber. The idea had been proposed previously by Leber ${ }^{3}$ and his colleagues but their evidence had not been accepted. At the time of the discovery of the aqueous veins the aqueous humour was still regarded as a stagnant fluid. ${ }^{4}$

Correspondence to: Professor Anders Bill, Department of Physiology and Medical Biophysics, BMC, Box 572, S-75123 Uppsala, Sweden.
There were, in fact, three good reasons for believing that the aqueous humour was stagnant. Firstly, there were no visible openings in the inner wall of Schlemm's canal that could drain the aqueous humour into the canal. Secondly, pressure measurements in the veins of the limbus region had indicated that the pressure there was the same or higher than in the anterior chamber. Thirdly, the hypothesis of Leber and his colleagues that the aqueous humour was a dynamic fluid had found no support in human studies and many of the animal experiments on which Leber based his opinion were considered rather crude. Thus, there were no pores permitting flow, the pressure gradient was in the wrong direction and no one had observed aqueous humour outflow in the limbus region in undisturbed eyes.

Ascher's observation immediately raised a number of questions about the normal pressure and flow conditions in the chambers of the eye and the outflow routes, and the mechanisms involved in the formation of the aqueous humour. It was also obvious that his concept would have a fundamental bearing on the theory of glaucoma.

Space does not allow a review of the ever-expanding literature concerning aqueous humour outflow. Therefore I will discuss here a few recent observations, mostly from my own laboratory, on the pressure conditions in the outflow routes, the nature of Schlemm's canal and two alternative routes for aqueous humour drainage, one physiological and one pathological. But first I will consider briefly the structure of the outflow routes.

\section{ANATOMY OF THE OUTFLOW ROUTES: THE PRESSURES}

Most of the aqueous humour that is drained in the chamber angle enters the intertrabecular spaces of the uveal meshwork. It then flows either towards Schlemm's canal or towards the connective tissue spaces that surround the muscle bundles of the ciliary muscle (Fig. 1). In monkeys under general anaesthesia these flow rates are similar. ${ }^{5,6}$ In humans the flow towards Schlemm's canal seems to be dominant. ${ }^{7}$ The intertrabecular spaces of the corneoscleral meshwork are relatively narrow but they do permit relatively large particles to pass. However, in the outermost 


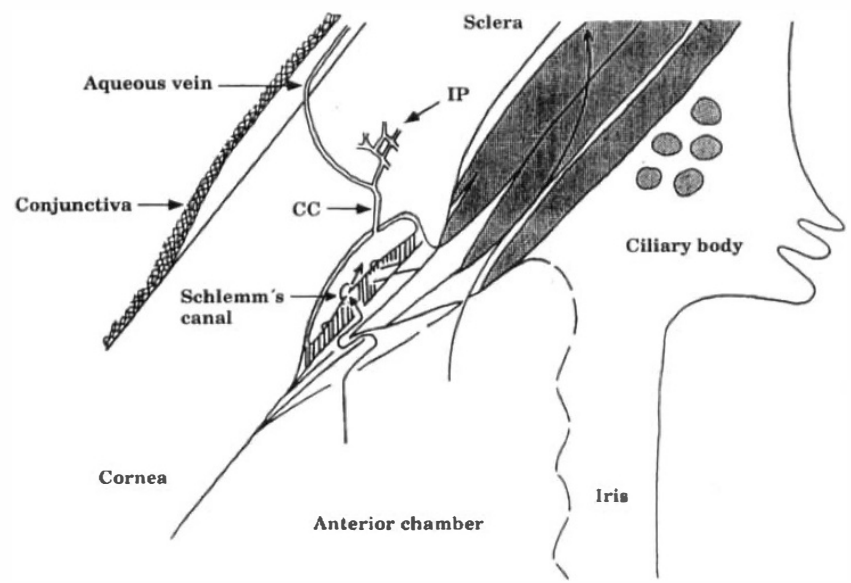

Fig. 1. In the human eye most of the aqueous humour flon's via the intertrabecular spaces and the jurtacanalicular tissue into Schlemm's canal via pores in the endothelial cells of the inner wall. The canal is drained by collector channels $(C C)$ into the intrascleral venous plexus (IP). Aqueous veins are rare due Io intrascleral mixing of aqueous humour and blood. Some aqueous humour flow's via the intertrabecular spaces and the connective tissue spaces between the muscle bundles of the ciliary muscle into the supraciliary and suprachoroidal spaces and out of the eye via the sclera. (From Bill and Mäepea." )

part of this meshwork and in the juxtacanalicular region the routes seem to be very narrow and even $0.1 \mu \mathrm{m}$ particles tend to accumulate here when perfused through the outflow routes ${ }^{8}$ (Fig. 1). Near the outer wall there are regions which seem empty on electron microscopy but do not permit tracers to enter, suggesting that they in fact contain a ground substance that does not stain for electron microscopy.

The endothelium of the inner wall of Schlemm's canal has one very remarkable feature that can be observed by light microscopy: so-called giant vacuoles. Electron microscopy has demonstrated that all the 'giant vacuoles' have at least one opening towards the juxtacanalicular region and that therefore they should be regarded as invaginations into the cells from that side. ${ }^{8}$ In some of these invaginations there is another remarkable feature, first described by Holmberg, ${ }^{9}$ of one or more transcellular

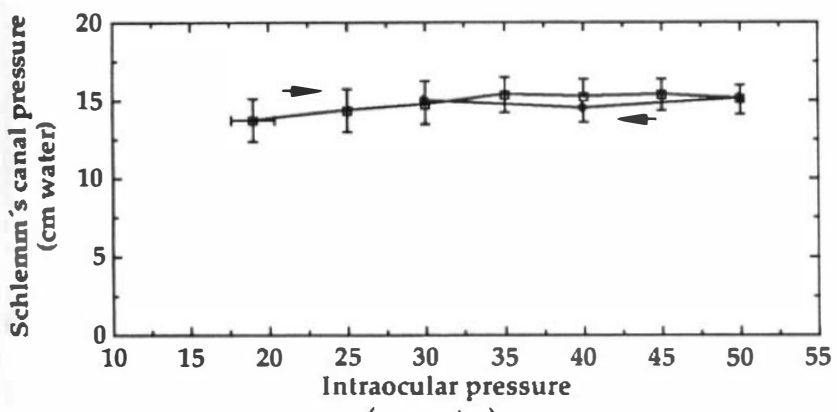

(cm water)

Fig. 2. The relationship between the intraocular pressure and Schlemm's canal pressure. An unfilled quadrant represents the change in Schlemm's canal pressure when the intraocular pressure was increased stepwise to $50 \mathrm{~cm} \mathrm{H}, \mathrm{O}$ and a filled rhomboid w'hen the intraocular pressure w'as decreased from 50 to $30 \mathrm{~cm} \mathrm{H}_{2} \mathrm{O}$. An unfilled quadrant and a rhomboid represent arithmetic means and the horizontal and vertical hars SE of the mean. (From Bill and Mäepea. ${ }^{31}$ ) pores permitting the flow of aqueous humour into Schlemm's canal. ${ }^{8-12}$

Early measurements of the pressure in Schlemm's canal performed by Perkins ${ }^{13}$ and Sears ${ }^{14}$ showed that the pressure was somewhat lower than the normal intraocular pressure (IOP) but, curiously, when the IOP was increased stepwise the pressure in the canal increased very much in parallel. Such behaviour indicated that most of the resistance - at least at higher pressures - was located in the collector channels and the venous system or that the cannula used for the measurements caused some damage to the tissue.

Improvements in the technique for measurements of the pressure in microvessels $\mathbf{s}^{15.16}$ have made it possible to measure the pressure in Schlemm's canal in monkeys. Fig. 2 shows the results of a recent study. ${ }^{17}$ The pressure in the canal is lower than the IOP at the spontaneous pressure. It is also clear that increments in pressure have only small effects on the pressure in the canal. From the flow and pressure data it can be calculated that about $90 \%$ of the outflow resistance is located between the anterior chamber and Schlemm's canal and that the resistance to flow from the canal to the episcleral veins is about $10 \%$. In these experiments the pressure in the canal was measured using a fine glass capillary with a tip diameter of less than $5 \mu \mathrm{m}$. Part of the sclera had to be trimmed to expose the outer wall of the canal.

The micropuncture technique was also used in attempts to measure the pressure in the juxtacanalicular region, but these measurements had to be performed blind. ${ }^{18}$ The cannula was pushed through the canal against the inner wall and then $10 \mu \mathrm{m}$ obliquely through the wall into the juxtacanalicular tissue. After measurement of the pressure there it was pushed $10 \mu \mathrm{m}$ deeper. The first measurement was thought to be $7 \mu \mathrm{m}$ from the inner wall, and the second $14 \mu \mathrm{m}$ from the wall. These experiments demonstrated that the main pressure drop at the spontaneous pressure occurred in the tissue 7-14 $\mu \mathrm{m}$ from the inner wall (Fig. 3). Interestingly when the IOP was stabilised at a pressure of about $15 \mathrm{mmHg}$ the region of high resistance moved towards the inner wall, so that the main resistance was within $7 \mu \mathrm{m}$ from the inner wall. Another interesting

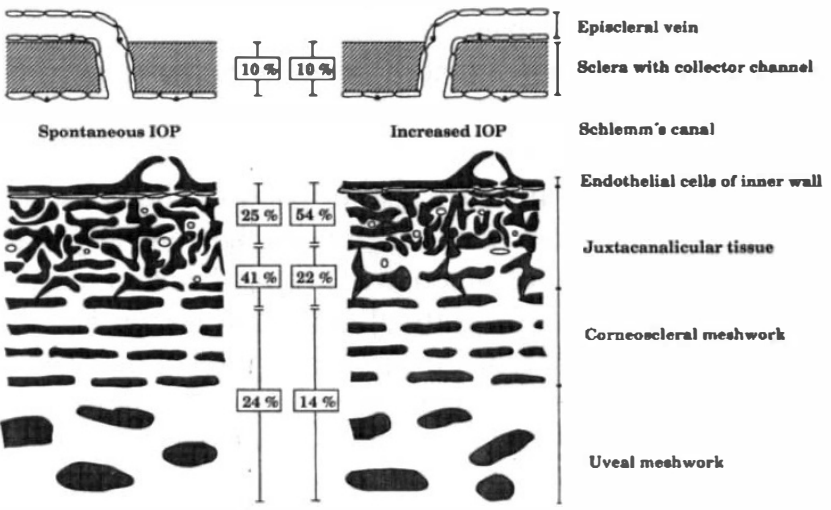

Fig. 3. The resistances, as percentages of the total, in different locations of the routes between the anterior chamber and the episcleral veins at spontaneous and increased intraocular pressure. (From Bill and Mäepea." 
observation was that all measurements of the pressure in the juxtacanalicular region gave similar results despite the fact that in some experiments the glass capillary was expected to have been near an invagination with a pore and in others some distance away from the nearest pore. This observation may be explained by the existence of preferential routes leading to pores, regions lacking pores having very little or no flow in the direction of the canal. An alternative explanation may be that in the juxtacanalicular meshwork the resistance to flow in the circumferential direction is much less than that to flow towards Schlemm's canal.

It is very unusual, of course, for the hydrostatic pressure outside a blood vessel to be higher than that inside the vessel. This requires that the blood vessel is prevented from collapsing, such as is the case for the sagittal venous sinus in the head. Schlemm's canal is prevented from collapse by the corneoscleral meshwork to which the inner wall of the canal is attached by the juxtacanalicular tissue. Another feature that may help to prevent collapse is the presence of septa in the canal. Such structures are quite common in some monkeys but rare in man.

A consequence of the higher pressure in the juxtacanalicular tissue than in the lumen of Schlemm's canal is that the endothelium of Schlemm's canal tends to be detached from the subendothelial tissue. Such detachment appears to be prevented to a large extent by a firm attachment. However, since it is not complete this attachment allows the invagination process to start in certain regions, often near the nucleus. One can speculate, of course, that too firm an attachment may prevent the formation of invaginations.

\section{THE PORES: POSSIBLE IMPORTANCE OF PLATELETS}

The pores of the inner wall of Schlemm's canal were detected with transmission electron microscopy, ${ }^{10}$ but this technique is very cumbersome when attempting to calculate the number of pores and estimate their sizes. For such studies scanning electron microscopy is much more useful. This technique has demonstrated that the number of pores in the inner wall of Schlemm's canal is of the order of 20000 in human eyes, ${ }^{12}$ with a similar number in monkeys. It has also shown that most of the pores seem to be located in the walls of the invaginations at some distance from the inlet into the invagination. Small pores can also be found occasionally in flat, thin parts of the cells and small openings seem to exist between the cells at places with incomplete tight junctions. ${ }^{19}$ It has been suggested that pinocytosis in thin parts of the cells may lead to the formation of membrane-covered minipores that eventually break and form small pores. ${ }^{8}$ In time these may increase in size. These observations have led to the question of why pores larger than $3 \mu \mathrm{m}$ are not found in the inner wall. It now seems likely that the answer to this question is that platelets tend to occlude such openings.

It was found in early studies with scanning electron microscopy that particles which might be platelets adhered to the inner wall of Schlemm's canal at some places. In more recent studies we have found that if the outflow routes are perfused with the chelating agents $\mathrm{Na}_{2}$ EDTA or $\mathrm{Na}_{2} \mathrm{EGTA},{ }^{20}$ large openings are created in the inner wall but that these tend to be quickly occluded by platelets. There seems to be occasional inflow of blood into the canal and platelet activation by contact with the subendothelial tissue. Such contact can be established, of course, also via large pores. An invagination with too large a pore will tend to collapse and then platelets may contact the subendothelial tissue via the pore. Thus, it is likely that an important role for the invaginations is to prevent contact between platelets and the subendothelial tissue. ${ }^{21}$ If openings in the inner wall of Schlemm's canal are made during treatment for glaucoma it may be important to prevent platelet activation; pretreatment with aspirin may therefore prove useful.

If it is true that the invaginations are important in preventing contact between platelets and the subendothelial tissue, then it should be possible to provoke platelet aggregation in large pores by reducing the IOP to a level that causes inflow of blood into the canal and collapse of the invagination. We performed such experiments some years ago in animals treated with alpha-chymotrypsin in one eye and heat-denaturated alpha-chymotrypsin in the other eye used as control. ${ }^{22}$ The pressure was increased to the normal level after 5 minutes of low pressure. Inspection of large areas of the inner wall of the control eye showed an interesting phenomenon (Fig. 4). Particles which seemed to be platelets had made contact with the subendothelial structure via a large pore. It appeared that the invagination had indeed collapsed as a result of the inversion of the pressure gradient and that under these conditions the platelets could contact the subendothelial tissue and attach to it.

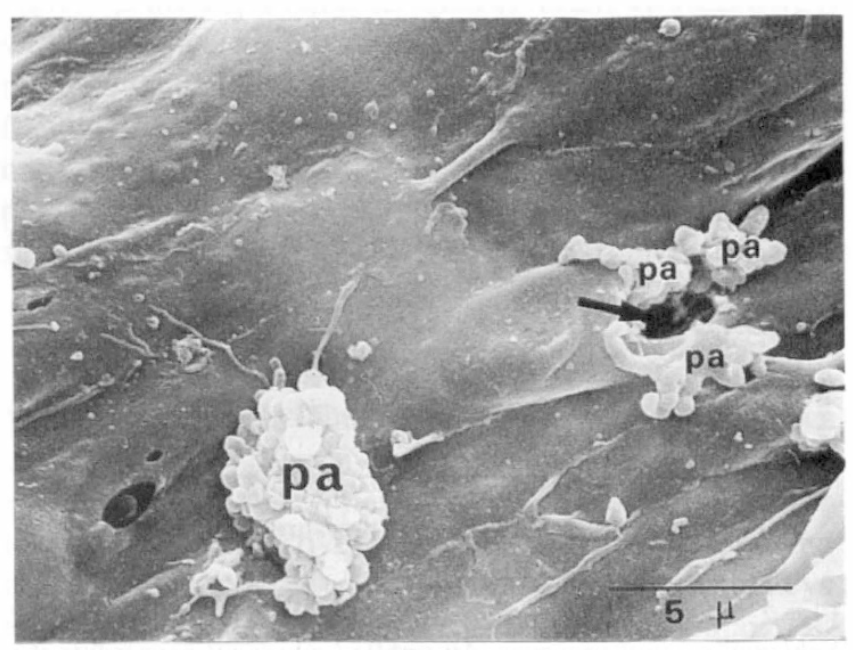

Fig. 4. Scanning electron micrograph of the inner wall of Schlemm's canal. The diameter of the pore indicated by the arrow was about $2.5 \mu \mathrm{m}$. Platelets were attached to the subendothelial tissue via the pore. There was no attachment of platelets to several smaller pores. pa, platelet aggregate. (Hamanaka and Bill, unpublished observations.) 


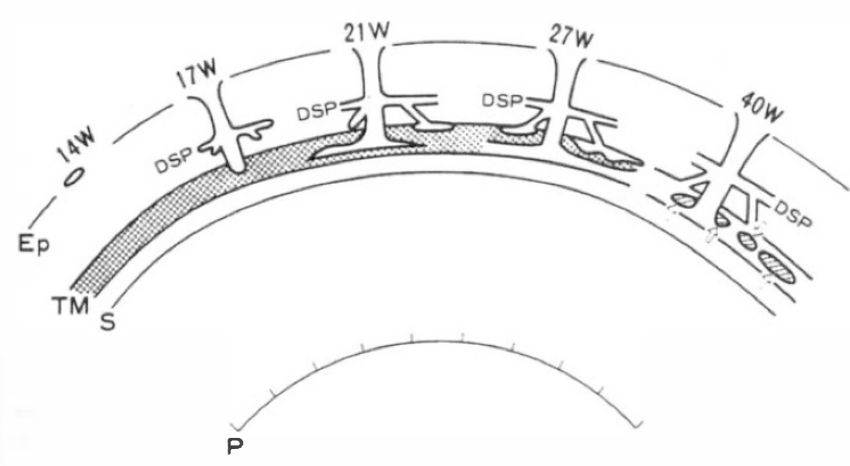

Fig. 5. The development of Schlemm's canal according to Hamanaka et al. ${ }^{23}$ Ep, episclera; TM, trabecular meshwork; $S$, Schwalbe's line; $P$, pupil; DSP, deep scleral plexus; $W$, weeks.

\section{THE DEVELOPMENT OF SCHLEMM'S CANAL}

One reason for the late detection of the aqueous veins was undoubtedly that most of the aqueous humour is mixed already with blood within the sclera; most of the collector channels drain aqueous humour into an intrascleral venous plexus, which is drained by the so-called anterior ciliary veins. The reason for this connection was elucidated in a recent study on the embryology of the canal of Schlemm ${ }^{23}$ performed on material obtained from Japan. It was found that at week 17 of gestation a deep scleral plexus had formed from extensions from episcleral vessels (Fig. 5). Extensions from the deep plexus appeared to grow towards the future corneoscleral meshwork and at the meshwork these blind extensions changed direction and started to grow circumferentially. They merged and formed an incomplete canal at about week 27, and at week 40 the canal was complete in most regions. We also looked for markers of endothelial cells and found factor-VIIIrelated antigen as well as Weibel-Palade bodies in the walls of the blood vessels and the outer wall of Schlemm's canal. In the inner wall factor-VIII-related antigen was unevenly distributed and Weibel-Palade bodies were scarce. The inner wall of Schlemm's canal thus retains the characteristics of a blood vessel despite its high degree of specialisation for aqueous humour drainage.

The embryology of the intrascleral venous plexus and Schlemm's canal may explain why the so-called anterior ciliary veins lack or have very few (and then only small) connections with the veins of the ciliary body. ${ }^{24}$

\section{CAN ENZYMES BE USED TO RINSE THE OUTFLOW ROUTES IN GLAUCOMA?}

Several studies have indicated that in open angle glaucoma there is an accumulation of some material in the outflow routes that appears to occlude the routes through the juxtacanalicular region. The reason for this is not at all clear. There may be a local production of this material or an inflow from some other region. Insufficient cleaning of the outflow routes due to ineffective phagocytosis by the trabecular cells may play a role. ${ }^{25}$ An obvious therapeutic approach would be to wash out the material from the juxtacanalicular region. Such a washout should probably start by dissolution of the material. Both $\mathrm{Na}_{2} \mathrm{EDTA}^{19}$ and alphachymotrypsin $^{21}$ have been found useful in washing out material that is normally present in the outflow routes. This is interesting, but what is needed is an enzyme that specifically attacks the pathological material and leaves the other structures intact. With access to eyes from autopsies of glaucoma patients it may be possible in the future to find the right substances for such therapy. Recent studies have indicated that the outflow routes are well maintained for many days under the right in vitro conditions ${ }^{26}$ and that even the pores can be studied under such conditions. ${ }^{27}$ It is thus feasible to perform experiments in which the conductance is determined in vitro both before and after treatment with enzymes.

One can speculate that once the right enzyme has been found by in vitro experiments on glaucomatous eyes a washout every $5-10$ years might be enough for pressure control in some cases of open angle glaucoma.

\section{OUTFLOW VIA UVEOSCLERAL ROUTES}

As mentioned above, it has been known for some time that in humans only a small part of the aqueous humour seems to be drained via the uveoscleral routes. ${ }^{7}$ The eyes that we were able to investigate using a direct method were tumour eyes from elderly patients. Attempts to calculate the uveoscleral flow in healthy humans have produced higher values. ${ }^{28}$ One way to increase the flow via the supraciliary and suprachoroidal spaces is of course to perform a cyclodialysis, which gives free access to the routes via the sclera. There are other, less dramatic ways. Thus, relaxation of the ciliary muscle caused by atropine enhances the flow between the muscle bundles, and recent studies with prostaglandin derivatives have suggested that there may also be other mechanisms by which the uveoscleral flow can be enhanced. ${ }^{29}$ The effect of prostaglandin treatment seems to be related to a change in the connective tissue of the ciliary muscle. ${ }^{30}$

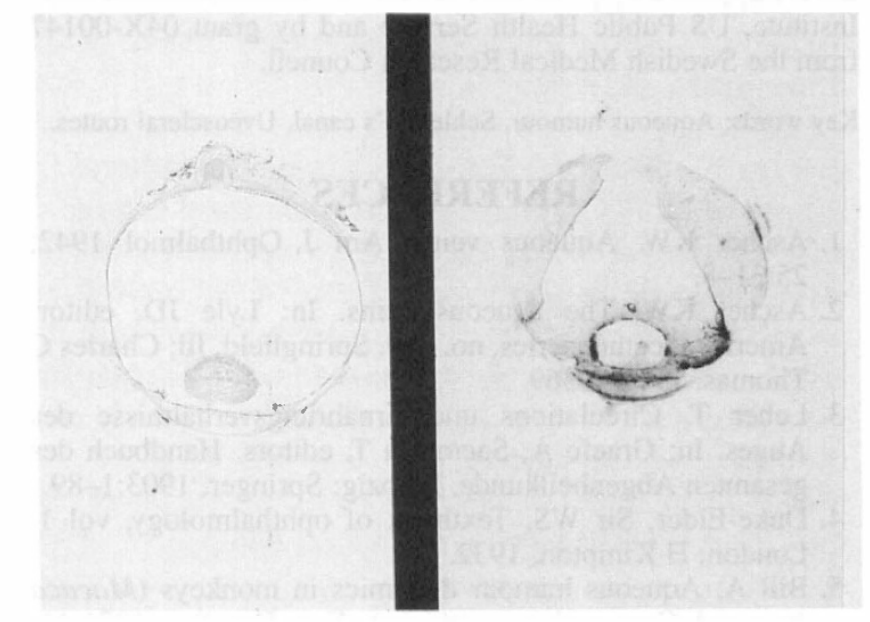

Fig. 6. Left: Section of a human eye with posterior synechia, cataract and retinal detachment. Right: Autoradiograph of the section. The anterior chamber was perfused with ${ }^{131}$ I-labelled human serum albumin which was washed out several hours later before enucleation of the eye. The eye was frozen and sectioned as described before. 


\section{OUTFLOW VIA THE VITREOUS CAVITY AFTER RETINAL DETACHMENT}

In most of our studies on uveoscleral flow we have injected or infused radioactively labelled proteins into the anterior chamber and then observed the distribution of labelled material in the eye. It has always been impressive to see what a perfect valve the iris is in preventing backflow into the posterior chamber. There was one exception to this rule. ${ }^{7}$ The patient had a posterior synechia and, as it turned out, also detachment of the retina. In this case we could see on autoradiographs how the labelled albumin injected into the anterior chamber had passed into the posterior chamber and from there into the vitreous cavity and, through a retinal hole, into the subretinal space (Fig. 6). The result of this experiment indicates that under conditions of retinal detachment there may be a highly pathological outflow of aqueous humour into the subretinal space and most probably into the choroid. It seems likely that there is some normal flow of aqueous humour from the posterior chamber via the vitreous cavity into the retina and out into the choroid. The magnitude of such a flow remains to be determined.

\section{THE FUTURE}

Since Ascher's discovery of the aqueous veins much has been learned about the anatomy, physiology and pharmacology of the outflow routes. ${ }^{31}$ Monkey eyes have proved very useful in many of these studies. It seems likely that in the near future we can expect very interesting results from in vitro perfused human eyes with or without glaucoma. ${ }^{32}$ For example, it will be possible to use the micropuncture technique mentioned above in determinations of the exact location of the excess outflow resistance in glaucomatous eyes and also to study the effects on outflow resistance of treatment with different enzymes.

I wish to thank Mr. Alf Johansson for expert technical assistance. Supported by grant RO1-EY00475 from the National Eye Institute, US Public Health Service and by grant 04X-00147 from the Swedish Medical Research Council.

Key words: Aqueous humour, Schlemm's canal, Uveoscleral routes.

\section{REFERENCES}

1. Ascher KW. Aqueous veins. Am J Ophthalmol 1942; 25:31-8.

2. Ascher KW. The aqueous veins. In: Lyle JD, editor. American lecture series, no. 403. Springfield, Ill; Charles C Thomas, 1961:1-369.

3. Leber T. Circulations und Ernährungsverhältnisse des Auges. In: Graefe A, Saemisch T, editors. Handbuch des gesamten Augenheilkunde. Leipzig: Springer, 1903:1-89.

4. Duke-Elder, Sir WS. Textbook of ophthalmology, vol 1. London: H Kimpton, 1932.

5. Bill A. Aqueous humour dynamics in monkeys (Macaca irus and Cercopithecus ethiops). Exp Eye Res 197.1;11: 195-206.

6. Bill A. Basic physiology of the drainage of aqueous humour. Exp Eye Res 1977;25:291-304.

7. Bill A, Phillips CI. Uveoscleral drainage of aqueous humour in human eyes. Exp Eye Res 1971;12:275-81.

8. Inomata H, Bill A, Smelser GK. Aqueous humour pathways through the trabecular meshwork and into Schlemm's canal in the Cynomolgus monkey (Macaca irus): an electron microscopic study. Am J Ophthalmol 1972;73:760-89.

9. Holmberg Å. Schlemm's canal and trabecular meshwork: an electron microscopic study of the normal structure in man and monkey (Cercopithecus ethiops). Doc Ophthalmol 1965;19:339-73.

10. Bill A. Scanning electron microscopic studies of the canal of Schlemm. Exp Eye Res 1970;10:214-8.

11. Bill A, Svedbergh B. Scanning electron microscopic studies of the trabecular meshwork and the canal of Schlemm: an attempt to localise the main resistance to outflow of aqueous humour in man. Acta Ophthalmol (Copenh) 1972;50: 295-320.

12. Rohen JW, Lütjen-Drecoll E. Morphology of aqueous outflow pathways in normal and glaucomatous eyes. In: Ritch R, Shields MB, Krupin T, editors. The glaucomas, vol I. St Louis, MO: Mosby, 1989:41-74.

13. Perkins ES. Pressure in the canal of Schlemm. Br J Ophthalmol 1955;39:215-9.

14. Sears ML. Pressure in the canal of Schlemm and its relation to the site of resistance of outflow of aqueous humour in the eyes of Ethiopian green monkeys. Invest Ophthalmol Vis Sci 1966;5:610-23.

15. Wiederhielm CA, et al. Pulsatile pressures in the microcirculation of frog's mesentery. Am J Physiol 1964;207:173-6.

16. Fein H. Microdimensional pressure measurements in electrolytes. J Appl Physiol 1972;32:560-4.

17. Mäepea O, Bill A. The pressure in the episcleral veins, Schlemm's canal and the trabecular meshwork in monkeys: effects of changes in intraocular pressure. Exp Eye Res 1989;49:645-63.

18. Mäepea O, Bill A. Pressures in the juxtacanalicular tissue and Schlemm's canal in monkeys. Exp Eye Res 1992;54: 879-83.

19. Epstein DL, Rohen JW. Morphology of the trabecular meshwork and inner wall after cationised ferritin perfusion in the monkey eye. Invest Ophthalmol Vis Sci 1991;32:160-71.

20. Hamanaka T, Bill A. Morphological and functional effects of $\mathrm{Na}_{2} \mathrm{EDTA}$ on the outflow routes for aqueous humour in monkeys. Exp Eye Res 1987;44:171-90.

21. Bill A, Mäepea O, Hamanaka T. Aspekte der Kammerwasserdrainage über den Schlemmschen Kanal. Klin Monatsbl Augenheilkd 1989;195:1-4.

22. Hamanaka T, Bill A. Effects of $\alpha$-chymotrypsin on the outflow routes for aqueous humour. Exp Eye Res 1988;46: $323-42$.

23. Hamanaka T, Bill A, Ichinohasama R, Ishida T. Aspects of the development of Schlemm's canal. Exp Eye Res 1992;55: 479-88.

24. Jocson VL, Grant WM. Interconnections of blood vessels and aqueous vessels in human eyes. Arch Ophthalmol 1965; 73:707-20.

25. Bill A. Basic physiology of the drainage of aqueous humour. Exp Eye Res 1977;25(Suppl):291-304.

26. Erickson-Lamy K, Rohen JW, Grant WM. Outflow facility studies in the perfused human ocular anterior segment. Exp Eye Res 1991;52:723-31.

27. Allingham RR, de Kater AW, Ethier CR, Anderson PJ, Hertzmark E, Epstein DL. The relationship between pore density and outflow facility in human eyes. Invest Ophthalmol Vis Sci 1992;33:1661-9.

28. Kaufman PL. Pressure-dependent outflow. In: Ritch R, Shields MB, Krupin T, editors. The glaucomas, vol I. St Louis, MO: CV Mosby, 1989:219-40.

29. Nilsson SFE, Samuelsson M, Bill A, Stjernschantz J. Increased uveoscleral outflow as a possible mechanism of ocular hypotension caused by prostaglandin $\mathrm{F}_{2 \alpha}-1$-isopropylester in the Cynomolgus monkey. Exp Eye Res 1989;48: 707. 
30. Lütjen-Drecoll E, Tamm E. Morphological study of the anterior segment of cynomolgus monkey eyes following treatment with prostaglandin $\mathrm{F}_{2 \alpha}$. Exp Eye Res 1988;47: 761-9.

31. Bill A, Mäepeao. Mechanisms and routes of aqueous humour drainage. In: Albert DM, Jakobiec FA, editors. The principles and practice of ophthalmology: the Harvard system. Philadelphia: WB Saunders (in press).

32. Kaufman PL. Pharmacologic trabeculocanalotomy. Arch Ophthalmol 1992;110:34-6. 